\title{
AQUISIÇÃO FONOLÓGICA EM CRIANÇAS COM ANTECEDENTES DE DESNUTRIÇÃO
}

\author{
Phonologic acquisition in children with \\ malnutrition antecedents
}

\author{
Luciana Melo de Lima ${ }^{(1)}$, Bianca Arruda Manchester de Queiroga ${ }^{(2)}$
}

\begin{abstract}
RESUMO
Objetivo: verificar a aquisição fonológica, por meio da análise dos processos fonológicos, de crianças de 2:1 a 6:6 anos com antecedentes de desnutrição. Métodos: foram estudadas 20 crianças, selecionadas através do registro de antecedentes de desnutrição dos prontuários médicos na Unidade de Saúde do Bairro Canafístula no município de Arapiraca-AL, onde também foram realizadas as avaliações de linguagem e das estruturas do sistema fonoarticulatório. Os participantes foram divididos por idade para facilitar a análise dos resultados. Resultados: foram encontrados sete processos fonológicos na fala das crianças avaliadas. Segundo a maioria da literatura referida, todos os processos encontrados nesta população, com exceção de dois processos, encontram-se fora do prazo esperado para serem eliminados. Conclusão: foi possível concluir que as crianças com antecedentes de desnutrição apresentavam, de modo geral, um retardo na aquisição fonológica.
\end{abstract}

DESCRITORES: Linguagem; Fala; Desnutrição; Desenvolvimento da Linguagem; Fonoaudiologia

\section{INTRODUÇÃO}

O indivíduo necessita de quantidades de energia e nutrientes obtidos através dos alimentos para que ocorra seu crescimento e desenvolvimento. Caso estas não sejam fornecidas corretamente, o organismo utilizará suas reservas que, se não repostas a tempo, proporcionarão uma deficiência nutricional caracterizando a desnutrição protéico-calórica (DPC) ${ }^{1}$. Este problema é causado por um complexo multifatorial e, em última instância, é determinado pelas condições de vida da população concernentes aos aspectos sociais e econômicos ${ }^{2}$.

O estado nutricional da criança pode ser representado pela avaliação de suas medidas antropométricas mediante valores individuais de peso e altura, segundo sexo e idade, tomando-se como referência padrões previamente definidos, sendo os índices altura/idade, peso/idade e peso/altura os mais freqüentemente empregados ${ }^{3}$.

(1) Fonoaudióloga; Clínica Otomed - AL; Especialista em Fonoaudiologia Hospitalar pela Universidade Estácio de Sá.

(2) Fonoaudióloga; Professora Adjunto do Curso de Fonoaudiologia da Universidade Federal de Pernambuco; Doutora em Psicologia Cognitiva pela Universidade Federal de Pernambuco.
A DPC acarreta graves e geralmente irreversíveis repercussões no Sistema Nervoso Central (SNC). O período de maior aceleração do crescimento cerebral estende-se desde a trigésima semana de gestação até, pelo menos, o final do segundo ano de vida; há, nesse período, maior probabilidade de risco de dano permanente ${ }^{4}$.

A má nutrição nos primeiros anos e meses de vida pode resultar em graves seqüelas ao indivíduo tais como: alterações no desenvolvimento cognitivo, mostrando um retardo significativo na aquisição da linguagem e na formação do conceito verbal. Se, além disso, as crianças foram pouco estimuladas, pode haver uma séria diminuição de seus coeficientes intelectuais, conseqüentemente dificuldade na aquisição da linguagem e maiores taxas de fracasso escolar ${ }^{5,6}$.

A linguagem é uma função superior do cérebro cujo desenvolvimento se sustenta em uma estrutura anatomofuncional geneticamente determinada e em estímulos verbais que lhe proporcionam o conteúdo ${ }^{6}$. Constitui um instrumento social usado em interações visando à comunicação. Pode ser definida como um sistema convencional de símbolos arbitrários que são combinados de modo sistemático e orientados para armazenar e trocar informações ${ }^{7}$.

Para que ocorra o desenvolvimento da linguagem humana, é necessário que existam bases orgâni- 
cas integras, bom funcionamento psíquico, ambiente social estimulador e inúmeros fatores cognitivolingüísticos. Sendo assim, a aquisição da linguagem consiste em um processo biopsicossocial complexo ${ }^{8}$, sendo o ambiente social determinante para que a criança desenvolva suas potencialidades por intermédio da imitação e do reforço às respostas apresentadas ${ }^{9}$.

Durante o desenvolvimento da linguagem oral, no período crítico compreendido entre o nascimento e a idade de 5 anos, acontece amadurecimento do conhecimento fonológico num processo gradativo, nãolinear e com variações individuais. O resultado desse desenvolvimento é o estabelecimento de um sistema fonológico condizente com o alvo-adulto, que é a fala do grupo social em que a criança está inserida ${ }^{10}$.

A compreensão da ocorrência de eventuais desordens fonológicas na fala da criança só será possível através da análise fonológica, que irá descrever as características formais dos padrões de pronúncia com desvio, baseando-se na Teoria da Fonologia Natural ${ }^{11}$. Esta teoria, afirma que a aquisição fonológica é um processo gradual de eliminação de processos mentais, naturais, universais e inatos, que são utilizados pela criança para facilitar aspectos mais complexos da fala ${ }^{10}$.

Ocorrem no desenvolvimento normal da criança os seguintes processos fonológicos e as idades previstas para eliminação: 1- redução de sílaba, quando há perda de uma das sílabas do vocábulo (2,6 anos); 2- harmonia consonantal, um fonema sofre a interferência de um vizinho que o antecede ou o segue $(2,6$ anos); 3- plosivação de fricativas, o modo de articulação dos fonemas fricativos é transformado em plosivo (2,6 anos); 4- simplificação da fricativa velar, quando a fricativa velar é omitida ou substituída (3,6 anos); 5posteriorização para velar, um fonema plosivo linguodental se transforma em um plosivo velar (3,6 anos); 6- posteriorização para palatal, há a alteração da zona de articulação transformando um fonema fricativo alveolar em um fonema fricativo palatal $(4,6$ anos); 7- frontalização de velares, um fonema plosivo velar transforma-se em plosivo linguo-alveolar (3 anos); 8- frontalização de palatal, o falante anterioriza a produção de uma consoante fricativa palatal, transformando-a geralmente numa fricativa alveolar (4,6 anos); 9- simplificação de líquidas, ocorre a substituição, semivocalização ou omissão das vibrantes (3,6 anos); 10- simplificação de encontro consonantal, o falante geralmente elimina um dos membros do encontro, em geral, a consoante líquida (7 anos); e 11- simplificação de consoante final, o falante elimina ou substitui a consoante final do vocábulo ( 7 anos $)^{12}$.

Ainda não se sabe ao certo os limites de eliminação desses processos durante a aquisição da fala, porém sabe-se que todos os processos fonológicos desaparecem em algum momento entre 1,6 e 4,0 anos e em algumas crianças podem demorar mais ${ }^{13}$.

Diante da relevância do tema abordado, este estudo justificou-se por oportunizar uma melhor compreensão do processo de aquisição fonológica em crianças com antecedentes de desnutrição, tanto para o fonoaudiólogo quanto para os demais profissionais envolvidos com o estudo do crescimento e desenvolvimento infantil.

O estudo teve como objetivo verificar a aquisição fonológica com base na teoria da fonologia natural, de crianças de 2,1 a 6,6 anos com antecedentes de desnutrição. De modo específico, o estudo pretendeu identificar a presença dos processos fonológicos na linguagem das crianças avaliadas e observar se a presença dos processos fonológicos apresentados pelas mesmas encontra-se dentro do esperado para suas faixas etárias.

\section{MÉTODOS}

O estudo foi realizado na Unidade de Saúde do Bairro Canafístula em Arapiraca-AL no mês de setembro de 2004. Foram avaliadas 22 crianças, na faixa etária da 2,1 a 6,6 anos, com antecedentes de desnutrição em qualquer grau.

Os critérios de inclusão foram faixa etária e o histórico clínico de desnutrição, obtido através do diagnóstico do pediatra. Foram excluídas do estudo crianças que apresentassem faixas etárias inferiores a 2,1 anos e superiores a 6,6 anos de idade; histórico de perdas auditivas; alterações miofuncionais orofaciais que pudessem interferir na produção da fala (distúrbios fonéticos); distúrbios sócio-emocionais e sintomatologia neurológica.

Depois da seleção dos participantes através dos prontuários médicos, estes foram contatados através dos agentes de saúde do Programa Saúde da Família os quais solicitaram que os mesmos fossem à Unidade de Saúde onde se realizaram os procedimentos para a coleta dos dados.

Os exames realizados foram: avaliação das estruturas do trato fonoarticulatório e avaliação fonológica através de um protocolo de avaliação intitulado Avaliação Fonológica da Criança (AFC) ${ }^{14}$.

As sessões foram realizadas individualmente, em uma sala na unidade de saúde. Tiveram duração média de 30 minutos e foram gravadas em fita cassete.

Para a análise fonológica, as crianças foram divididas em seis grupos de acordo com as faixas etárias: G1 (2,1 a 3,0 anos); G2 (3,1 a 3,6 anos); G3 (4,0 a 4,6 anos); G4 (4,7 a 5,0 anos); G5 (5,1 a 5,6 anos) e G6 (6,1 a 6,6 anos) e apresentadas em forma tabular.

Os grupos ficaram com as respectivas idades: G1 $(2,1$ a 3,0 anos $\cong 2,5) ; G 2$ (3,1 a 3,6 anos $\cong 3,3) ;$ G3 $(4,1$ a 4,6 anos $\cong 4,2) ;$ G4 $(4,7$ a 5,0 anos $\cong$ 4,8); G5 (5,1 a 5,6 anos $\cong 5,4)$ e G6 (6,1 a 6,6 anos $\cong 6,5)$. 
Durante o período de coleta de dados não foi possível encontrar crianças na faixa etária de 3,7 a 4,0 anos.

A pesquisa passou pelos critérios de avaliação do Comitê de Ética em pesquisa da Universidade Federal de Pernambuco, tendo sido aprovada com o número 190/04.

Foi criado um banco de dados utilizando o software estatístico SPSS 10.0, tendo sido realizada análises estatísticas descritivas.

\section{RESULTADOS}

Serão apresentados os processos encontrados em cada grupo de idade.

$\mathrm{Na}$ Tabela 1 observam-se os grupos que apresentaram o processo de redução de sílaba. Este processo foi observado nas crianças com faixas etárias até 5,6 anos, sendo vistas em $50 \%$ das crianças do estudo.

O processo de simplificação de fricativa velar encontrado em algumas crianças do estudo está detaIhado na Tabela 2. É possível observar que apesar de não ter mais aparecido nas crianças do G4, as quais possuem até 5,0 anos de idade, aparece em $50 \%$ das crianças do G5.

A Tabela 3 mostra o que foi encontrado em relação aos processos de posteriorização para palatal e frontalização de palatal. Este processo foi visto em apenas duas crianças do estudo, uma do G1 e uma do G2.

O processo de simplificação de líquidas foi observado em muitas crianças do estudo como está descrito na Tabela 4. Das 20 crianças analisadas, 15 mantiveram este processo (75\%). Todas as crianças do G6 que têm idade entre 6,0 e 6,6 anos apresentaram-no.

$\mathrm{Na}$ Tabela 5 estão descritos os dados referentes ao processo de simplificação de encontro consonantal o qual foi visto em $100 \%$ das crianças do estudo.

A Tabela 6 apresenta os dados referentes ao processo de simplificação da consoante final. Com estes pode-se observar que até o G3 ele foi encontrado em $100 \%$ das crianças. Este processo foi visto em $85 \%$ das crianças do estudo.

Os outros processos que foram investigados (harmonia consonantal, plosivação de fricativas, posteriorização para velar e frontalização de velares) não ocorreram na população estudada.

Tabela 1 - Distribuição da freqüência absoluta e relativa da ocorrência do processo de redução de sílaba nos grupos etários

\begin{tabular}{lll}
\hline \multicolumn{1}{c}{ Grupos } & $\mathrm{F}$ & $\%$ \\
\hline Grupo 1 $(\mathrm{N}=3)$ & 3 & 100 \\
Grupo 2 $(\mathrm{N}=4)$ & 2 & 50 \\
Grupo 3 $(\mathrm{N}=4)$ & 1 & 25 \\
Grupo 4 $(\mathrm{N}=3)$ & 2 & 66,6 \\
Grupo 5 $(\mathrm{N}=4)$ & 2 & 50 \\
Grupo 6 $(\mathrm{N}=2)$ & 0 & 0 \\
\hline Total $(\mathrm{N}=\mathbf{2 0})$ & $\mathbf{1 0}$ & $\mathbf{5 0}$ \\
\hline
\end{tabular}

$\mathrm{N}=$ Número de participantes, $\mathrm{F}=$ Freqüência Absoluta 
Tabela 2 - Distribuição da freqüência absoluta e relativa da ocorrência do processo de simplificação de fricativa velar nos grupos etários

\begin{tabular}{lll}
\hline Grupos & F & $\%$ \\
\hline Grupo 1 $(\mathrm{N}=3)$ & 3 & 100 \\
Grupo 2 $(\mathrm{N}=4)$ & 3 & 75 \\
Grupo $3(\mathrm{~N}=4)$ & 1 & 25 \\
Grupo $4(\mathrm{~N}=3)$ & 0 & 0 \\
Grupo $5(\mathrm{~N}=4)$ & 2 & 50 \\
Grupo $6(\mathrm{~N}=2)$ & 0 & 0 \\
\hline Total $(\mathrm{N}=\mathbf{2 0})$ & $\mathbf{9}$ & $\mathbf{4 5}$ \\
\hline N= Número de participantes, F = Freqüência Absoluta &
\end{tabular}

Tabela 3 - Distribuição da freqüência absoluta e relativa da ocorrência dos processos de posteriorização para palatal e frontalização de palatal nos grupos etários

\begin{tabular}{lll}
\hline Grupos & F & $\%$ \\
\hline Grupo 1 $(\mathrm{N}=3)$ & 1 & 33,33 \\
Grupo 2 $(\mathrm{N}=4)$ & 1 & 25 \\
Grupo $3(\mathrm{~N}=4)$ & 0 & 0 \\
Grupo $4(\mathrm{~N}=3)$ & 0 & 0 \\
Grupo 5 $(\mathrm{N}=4)$ & 0 & 0 \\
Grupo 6 $(\mathrm{N}=2)$ & 0 & 0 \\
\hline Total $(\mathrm{N}=\mathbf{2 0})$ & 2 & 10 \\
\hline
\end{tabular}

$\mathrm{N}=$ Número de participantes, $\mathrm{F}=$ Freqüência Absoluta 
Tabela 4 - Distribuição da freqüência absoluta e relativa da ocorrência do processo de simplificação de líquidas nos grupos etários

\begin{tabular}{lll}
\hline Grupos & F & $\%$ \\
\hline Grupo 1 $(\mathrm{N}=3)$ & 3 & 100 \\
Grupo 2 $(\mathrm{N}=4)$ & 3 & 75 \\
Grupo 3 $(\mathrm{N}=4)$ & 3 & 75 \\
Grupo $4(\mathrm{~N}=3)$ & 2 & 66,6 \\
Grupo $5(\mathrm{~N}=4)$ & 2 & 50 \\
Grupo $6(\mathrm{~N}=2)$ & 2 & 100 \\
\hline Total $(\mathrm{N}=20)$ & 15 & 75 \\
\hline
\end{tabular}

$\mathrm{N}=$ Número de participantes, $\mathrm{F}=$ Freqüência Absoluta

Tabela 5 - Distribuição da freqüência absoluta e relativa da ocorrência do processo de simplificação de encontro consonantal nos grupos etários

\begin{tabular}{ccc}
\hline Grupos & F & $\%$ \\
\hline Grupo 1 $(\mathrm{N}=3)$ & 3 & 100 \\
Grupo 2 $(\mathrm{N}=4)$ & 4 & 100 \\
Grupo 3 $(\mathrm{N}=4)$ & 4 & 100 \\
Grupo $4(\mathrm{~N}=3)$ & 3 & 100 \\
Grupo $5(\mathrm{~N}=4)$ & 4 & 100 \\
Grupo $6(\mathrm{~N}=2)$ & 2 & 100 \\
\hline Total $(\mathrm{N}=\mathbf{2 0})$ & $\mathbf{2 0}$ & 100 \\
\hline
\end{tabular}

$\mathrm{N}=$ Número de participantes, $\mathrm{F}=$ Freqüência Absoluta 
Tabela 6 - Distribuição da freqüência absoluta e relativa da ocorrência do processo de simplificação de consoante final nos grupos etários

\begin{tabular}{lll}
\hline Grupos & F & $\%$ \\
\hline Grupo 1 $(\mathrm{N}=3)$ & 3 & 100 \\
Grupo $2(\mathrm{~N}=4)$ & 4 & 100 \\
Grupo $3(\mathrm{~N}=4)$ & 4 & 100 \\
Grupo $4(\mathrm{~N}=3)$ & 2 & 66,6 \\
Grupo 5 $(\mathrm{N}=4)$ & 3 & 75 \\
Grupo 6 $(\mathrm{N}=2)$ & 1 & 50 \\
\hline Total (N=20) & 17 & $\mathbf{8 5}$ \\
\hline $\mathrm{N}=$ Número de participantes, F = Freqüência Absoluta
\end{tabular}

\section{DISCUSSÃO}

Ao realizar um paralelo entre o que foi encontrado nas crianças da pesquisa e o que refere a literatura podese observar que o processo de redução de sílaba mostrou-se presente nas crianças com até 5,6 anos de idade, tendo oscilado nas crianças com idade inferiores, de $25 \%$ a $100 \%$. Na literatura, observa-se que as crianças eliminam este processo em torno dos 3,6 anos ${ }^{15}$, outros autores afirmam que isso se dá até os três anos ${ }^{16}$. Porém, um estudo realizado com crianças de 2,1 a 3,0 anos de idade, constatou que essa população ainda não havia eliminado o processo de redução de sílaba ${ }^{13}$. Deste modo, pode-se afirmar que este processo já deveria ter sido eliminado pelas crianças dos grupos $3,4 \mathrm{e}$ 5 , onde, contudo, ainda seja observado.

No caso do processo de simplificação de fricativa velar, todas as crianças com idades de 2,1 a 3,0 anos apresentaram-no, mas sua freqüência foi diminuindo com o avanço da idade e as crianças do G4 (4,7 a 5,0 anos) eliminaram-no. Houve um reaparecimento em 50\% das crianças com faixa etária entre 5,1 e 5,6 anos, porém em nenhuma criança com mais de 6,0 anos foi constatado. Com relação a este processo, há referências na literatura que por volta dos três anos de idade a simplificação de velares é eliminada ${ }^{13,15,16}$. Além disso, um outro estudo constatou que os fonemas fricativos são adquiridos em torno dos quatro anos ${ }^{17}$. E levando-se em consideração esta constatação, este processo deveria ter sido eliminado nas crianças a partir do G3 no presente estudo.
Os processos de posteriorização para palatal e frontalização de palatal só foram vistos no G1 e G2 que compreendem crianças de até 3,6 anos. Alguns autores defendem que esses processos são eliminados aos 4,6 anos ${ }^{12}$. Outros afirmam que a frontalização e a posteriorização desaparecem por volta dos 4,0 anos. A frontalização é mais freqüente até os três anos, mas pode ocorrer até por volta dos 4,6 anos ${ }^{15}$. Esses processos foram produzidos por crianças além de três anos de idade em outros estudos ${ }^{16}$. Deste modo, a ocorrência destes processos no grupo estudado encontra-se dentro da normalidade, pois ocorreu apenas na faixa etária esperada.

Com relação ao processo de simplificação das líquidas, pode-se observar que $100 \%$ das crianças com 2,1 a 3,0 anos e as com 6,0 a 6,6 anos apresentaram este processo. Para alguns autores, as consoantes líquidas possuem um domínio complexo e tardio em distintos sistemas lingüísticos ${ }^{18}$. A literatura também refere que aos quatro anos ocorre a produção normal das líquidas ${ }^{13}$, ou que este processo é eliminado mais ou menos aos 3,6 anos ${ }^{15}$. Além disso, autores afirmam que a simplificação de líquidas é o processo mais freqüente entre as crianças de 3,1 a 5,6 anos de idade ${ }^{13}$. Assim, pode-se afirmar que no presente estudo este processo não deveria mais ser encontrado nas crianças a partir do G3.

Os dados encontrados nesta pesquisa mostram que as crianças até os 6,6 anos ainda não eliminaram o processo de simplificação de encontro consonantal. Segundo a literatura, este é o processo 
mais duradouro, desaparecendo aos quatro anos ${ }^{13}$, podendo aparecer até os cinco anos ${ }^{15}$. Em relação a esses autores, as crianças estudadas apresentariam um retardo na eliminação deste processo. Porém outro estudo realizado com crianças de três a sete anos, refere que crianças eliminam este processo aos 7 anos ${ }^{16}$. Também é importante ressaltar que aquisição do encontro consonantal é muito influenciada pelo meio sociocultural, já que é freqüente o modelo inadequado deste encontro em nível social mais baixo.

Observou-se, com relação ao processo de simplificação de consoante final (Tabela 6), que todas as crianças até os 4,6 anos apresentaram-no. Após esta idade houve oscilações e aos 6,6 anos $50 \%$ das crianças ainda possuíam-no. Alguns estudos relatam que as crianças apresentam este processo além dos três anos, sendo este eliminado em torno dos sete anos. Outros afirmam que o processo de simplificação da consoante final desaparece até mais ou menos quatro anos ${ }^{16}$.

Confrontando os resultados com a maioria da literatura referida, é possível afirmar que todos os processos encontrados nesta população, com exceção dos processos de posteriorização para palatal e frontalização de palatal, encontram-se fora do prazo esperado para serem eliminados. Mas, não se pode deixar de levar em consideração que para uma correta emissão dos fonemas, é necessário o aprendizado dos sons que compõem as palavras e isto depende de modelos adequados fornecidos pelos falantes do meio em que o indivíduo está inserido. As crianças inseridas em meios socioculturais onde há dificuldades em trocas com os adultos tendem a apresentar desvios fonológicos mais acentuados.

Os dados obtidos nesse estudo são de extrema importância, pois foram observados atrasos da eliminação de alguns processos fonológicos na popula- ção estudada, mesmo quando se considerou os autores que propuseram idades mais avançadas para que esta eliminação ocorresse ${ }^{13,15,16}$.

Sabe-se que o atraso na eliminação destes processos pode trazer sérias alterações na aquisição da leitura e escrita nas crianças que estão em idade de alfabetização, pois existe uma combinação dos fenômenos biológicos e ambientais no aprendizado da linguagem escrita, que envolve a integridade motora, a integridade sensório-perceptual e a integridade socioemocional (possibilidades reais que o meio oferece em termos de quantidade, qualidade e freqüência de estímulos). Além disso, o domínio da linguagem também é um fator importante no desenvolvimento do aprendizado da leitura e da escrita ${ }^{19}$.

A desnutrição é acima de tudo um problema social, que acomete predominantemente indivíduos de níveis sociais baixos. Sendo assim, percebe-se que as condições de vida e o meio sócio-cultural em que estes indivíduos vivem são desfavoráveis para a abrangência da linguagem, no que se refere ao alcance do padrão fonológico da língua.

\section{CONCLUSÃO}

Diante das informações referidas, pode-se concluir que foi observada a presença de sete processos fonológicos na linguagem da população analisada e que de acordo com a literatura, apenas as crianças de faixa etária entre 2,6 e 3,0 anos apresentavam os processos correspondentes a suas idades; o restante apresentou atraso na eliminação dos processos.

Os dados encontrados no presente estudo apontam para a repercussão da desnutrição no crescimento e desenvolvimento infantil, reforçando a necessidade de políticas de saúde mais efetivas no que refere ao combate da desnutrição infantil.

\section{ABSTRACT}

Purpose: to verify the phonologic acquisition, through the analysis of the phonologic process, in children with age from 2-1 to 6-6 years with malnutrition antecedents. Methods: 20 children, selected through the malnutrition antecedent register of medical handbooks in the Unit of Health of Canafístula Neibourhood had been studied in the city of Arapiraca-AL, where the evaluations of language and the structures of the factory system had been also carried through. The participants had been divided by age to facilitate the analysis of the results. Results: 7 phonologic processes was found in the speech of the evaluated children. According to the majority of related literature, all the processes found in this population, with exception of two process, are outside the waited period stated for being eliminated. Conclusion: it was possible to conclude that children with antecedents of malnutrition showed, generally speaking, a retardation in the phonologic acquisition.

KEYWORDS: Language; Speech; Malnutrition; Language Development; Speech, Language and Hearing Sciences 


\section{REFERÊNCIAS}

1. Nóbrega FJ. Desnutrição intra-uterina e pós-natal. São Paulo: Panamed; 1981.

2. Schmitz BAS, Picanço MR, Aquino KKNC, Bastos J, Giorgini E, Cardoso R et al. Prevalência da desnutrição e anemia em pré-escolares de Brasília Brasil. Pediatr Mod. 1998; 34(4):155-64.

3. Rissin A. Estado nutricional de crianças menores de cinco anos: uma análise epidemiológica no Brasil e, especialmente no Nordeste, como referência para a fundamentação de programas de intervenção nutricional [Mestrado]. Recife (PE): Instituto Materno Infantil de Pernambuco; 1997. 4. Guardiola A, Egewarth C, Rotta NT. Avaliação do desenvolvimento neuropsicomotor em escolares de primeira série e sua relação com o estado nutricional. J Pediatr. 2001; 77(3):189-96.

5. Onis M, Frongillo EA, Blössner M. ¿Está disminuyendo la malnutrición? Análisis de la evolución del nivel de malnutrición infantil desde 1980. Boletin de la Organización Mundial de la Salud. Recopilación de articulos. 2001; n. 4.

6. Castaño J. Bases neurobiológicas del lenguaje y sus alteraciones. Rev Neurol. 2003; 8(36):781-5.

7. Nogueira S, Fernández B, Porfírio H, Borges L. A criança com atraso na linguagem. Saúde Infantil. 2000; 22(1):5-16.

8. Issler S. Articulação e linguagem. Rio de Janeiro: Lovise; 1996.

9. Carvalho JF, Homem FCB. A influência do meio na aquisição da linguagem. Fono Atual. 2001; 17:14-6. 10. Lamprecht RR. Sobre os desvios fonológicos. In:
Lamprecht RR, et al. Aquisição fonológica do português: perfil do desenvolvimento e subsídios para terapia. Porto Alegre: Artmed; 2004.

11. Mota HB. Terapia fonoaudiológica para os desvios fonológicos. Rio de Janeiro: Revinter; 2001.

12. Wertzner HF. Distúrbio fonológico. In: Andrade $\mathrm{CMF}$, Marcondes E. Fonoaudiologia em pediatria. São Paulo: Sarvier; 2003.

13. Wertzner HF, Galea DES, Almeida RC. Uso do processo fonológico de simplificação de velar em crianças de 2;1 a 3;0 anos de idade. J Bras Fonoaudiol. 2001;2(8):233-88.

14. Yavas M, Hernandorene CM, Lamprech RR. Avaliação fonológica da criança: reeducação e terapia. Porto Alegre: Artes Médicas; 2001.

15. Yavas M. Padrões de aquisição da fonologia do português. Letras Hoje. 1988; 23:7-30.

16. Wertzner HF. Estudo da aquisição do sistema fonológico: uso de processos fonológicos em crianças de três a sete anos. Pró-Fono R Atual Cient. 1995; 7(1):21-6.

17. Wertzner HF, Carvalho IAM. Ocorrência de "erros" nos fonemas fricativos durante o processo de aquisição do sistema fonológico. J Bras Fonoaudiol. 2000; 1(2):67-74.

18. Hernandorena CLM, Lamprecht RR. Aquisição das consoantes líquidas do português. Letras Hoje. 1997; 32:7-22.

19. Pinheiro AMV. Dificuldades específicas de leitura: a identificação de déficits cognitivos e a abordagem do processamento de informação. Psicol Teor Pesq. 1995; 11(2):107-15.

RECEBIDO EM: 20/11/06

ACEITO EM: 21/02/07

Endereço para correspondência:

Rua Professor Arthur de Sá, S/N;

Departamento de Fonoaudiologia - UFPE Cidade Universitária

Recife - PE

CEP: $50670-420$

Tel: (81) 21268927

E-mail: lucianamlima@hotmail.com 